\title{
Slow and intermittent stress relaxation of biomass granular media
}

\author{
Florent Thevenon ${ }^{1,2,3^{*}}$, Bharath Devulapalli ${ }^{2}$, Muriel Marchand ${ }^{1}$, Thierry Melkior ${ }^{1}$, and Alain de Ryck ${ }^{2}$ \\ ${ }^{1}$ Université Grenoble Alpes, CEA Liten, DTBH, 17 avenue des Martyrs, Grenoble Cedex 09, 38054, France \\ ${ }^{2}$ Centre RAPSODEE, UMR CNRS 5302 - IMT Mines Albi, Campus Jarlard, Albi Cedex 09, 81013, France \\ ${ }^{3}$ Agence de l'Environnement et de la Maîtrise de l'Energie, 20 avenue du Grésillé- BP 90406, Angers Cedex 01, 49004, France
}

\begin{abstract}
In the present work, the relaxation of several wood powders has been investigated in an annular shear cell. It is found that the slow logarithmic relaxation commonly observed for various materials including granular materials is interrupted by large events. Their frequency and amplitude are investigated with respect to particle size and stress-history by measuring stresses and strain. These large events during the relaxation appear to be controlled by the deformation. The coarser the particles are, the bigger is the deformation of the powder bed between two fast relaxation events and during the event.
\end{abstract}

\section{Introduction}

The logarithmic relaxation of compressed materials has been already evidenced for various materials like metals and granular matter [1]. The same behaviour is observed in SHS tests (Shear-Hold-Shear) in solid friction [2] or granular friction [3].

In solid or granular media, this behaviour may be described by a rate-and-state friction [4-5]. More recently, a model with an assembly of parallel exponential relaxers with different relaxation times (DRT) has been introduced and then further developed to describe logarithmic and non-monotonic relaxations [6]. This latter model allows a good description of nonmonotonic relaxation experiments with granular media [7].

Here, we present some experimental data displaying a nonmonotonic behaviour of relaxing wood powders in Shear and Hold (SH) tests and in oedometric compression tests. The study of the rheology of fine biomass powders is gaining interest over the last few years, due to their applications in thermochemical processes such as gasification [8-9] or combustion [10]. Their flowability is crucial for a controlled conversion, but their fibrous nature and low apparent specific mass do not favour gravitational flows and may induce aging phenomena during storage.

\section{Materials and methods}

\subsection{Materials}

The powders used in this work are obtained by milling resinous wood chips, torrefied (thermally treated during 55 minutes at $250^{\circ} \mathrm{C}$ under neutral atmosphere) or not.
Different mills and various sieving screens have been used to produce powders having different characteristics: - A coarse powder of torrefied wood (Tor5), ground with a knife mill equipped with a $5 \mathrm{~mm}$ screen and sieved with a $1 \mathrm{~mm}$ screen.

- A coarse powder of raw wood (Raw5), ground with a knife mill equipped with a $5 \mathrm{~mm}$ screen and sieved with a $1 \mathrm{~mm}$ screen.

- A powder of raw wood ground in a vibration mill (RawVib) and sieved with a $1 \mathrm{~mm}$ screen.

- A fine powder of torrefied wood (Tor1), ground with a knife mill equipped with a $1 \mathrm{~mm}$ screen.

- A fine powder of raw wood (Raw1fine), ground with a knife mill equipped with a $1 \mathrm{~mm}$ screen and sieved with a $200 \mu \mathrm{m}$ screen.

\subsection{Methods}

\subsubsection{Particle and bulk characterizations}

A dynamic image analyser (Camsizer XT, RETSCH) has been used to characterize the particle size and aspect ratio (defined as the projected particle width over its length) distributions. The size and shape descriptors are respectively the median of the minimum Feret diameter and aspect ratio distributions, respectively named $\mathrm{d}_{50}$ and $\mathrm{AR}_{50}$.

The particle density $\rho_{\text {particle }}$ has been measured for all the samples by water pycnometry.

The descriptors of these wood powders are listed in Table 1. The particles are quite elongated, although the smallest ones are rounder. The grinding of torrefied wood has resulted in finer particles than raw wood, with nearly similar shape.

\footnotetext{
* Corresponding author : florent.thevenon@mines-albi.fr

A video is available at https://doi.org/10.48448/j300-5254
} 
The surface state of raw and torrefied wood differ, due to the Maillard reactions but the apparent bulk coefficients of friction of these samples are mainly the same. This friction coefficient $\mu$ has been measured in the apparatus described in the following paragraph.

Initial loose packing characteristics-Table 1 also displays the bulk loose density $\rho_{\text {bulk }}$ and initial bed porosity $\varepsilon$. The wood powders studied in this work are made of elongated particles and have a high initial porosity compared to the random loose packing of monodisperse spheres which is about 0.52 .

Table 1. Size and shape descriptors of the wood powders and bulk properties.

\begin{tabular}{|l|l|l|l|l|l|l|}
\hline Powder & $\begin{array}{c}\mathrm{d}_{50} \\
\mu \mathrm{m}\end{array}$ & $\mathrm{AR}_{50}$ & $\begin{array}{c}\rho_{\text {particle }} \\
\mathrm{g} / \mathrm{cm}^{3}\end{array}$ & $\begin{array}{c}\rho b u l k \\
\mathrm{~g} / \mathrm{cm}^{3}\end{array}$ & $\varepsilon$ & $\mu$ \\
\hline Raw5 & 662 & 0.37 & 0.83 & 0.16 & 0.81 & 1.01 \\
\hline Tor5 & 264 & 0.43 & 0.60 & 0.17 & 0.71 & 0.92 \\
\hline RawVib & 220 & 0.64 & 1.03 & 0.30 & 0.71 & 0.85 \\
\hline Tor1 & 25 & 0.61 & 0.83 & 0.20 & 0.76 & 0.92 \\
\hline Raw1fine & 25 & 0.64 & 0.99 & 0.12 & 0.88 & 0.81 \\
\hline
\end{tabular}

\subsubsection{Relaxation testing}

The relaxation behaviour of the wood powders has been studied with a Schulze Ring Shear Tester [11]. Two sizes of the annular cell have been used, a big cell (inner and outer diameters of 5 and $10 \mathrm{~cm}$ ), and a small cell ( 3 and 6 $\mathrm{cm}$ respectively).

The shear stress is measured thanks to two load beams, each of them being connected to a tie rod with the same lever arm for both cells. During the shearing, the tie rods prevent the lid from rotating and transfer the tensile force to the load beams. A digital displacement indicator is used for the measurement of the lid, giving the height of the bulk solid sample.

The sample is poured uniformly in the cell without applying force onto the surface to obtain a homogeneous porosity inside the sample. The powder surface is erased with a spatula and the sample is weighted to determine its initial bulk density $\rho_{\text {bulk }}$ and porosity $\varepsilon$ (Table 1 ).

The relaxation test is a four-step procedure, as illustrated by Figure 1.

First, a normal load is applied to the lid and the sample is sheared until steady flow at a rotational velocity of 0.9 $\mathrm{mrad} / \mathrm{s}$. Once the shear stress and the powder height are constant, the rotation of the cell is stopped and hold during 15 to 30 minutes depending on the experiment. In this time period, both the shear stress and the sample height relax. Finally, the shear stress is removed by reversing the rotation of the cell during a few seconds. The system relaxes its height about 20 minutes more with only the normal loading. Both the height and the shear stress are recorded during the whole procedure.

The behaviour of the powders during the relaxation has been studied under normal stresses $\sigma$ varying from 0.9 to $10.5 \mathrm{kPa}$ in the big cell, and from 2.7 to $30.0 \mathrm{kPa}$ in the small cell. It is typically the range of stresses applied on a powder at the bottom of an industrial hopper. Experiments have been performed by increasing the normal load so that the powder bed is considered to be in a loose state before the experiment.

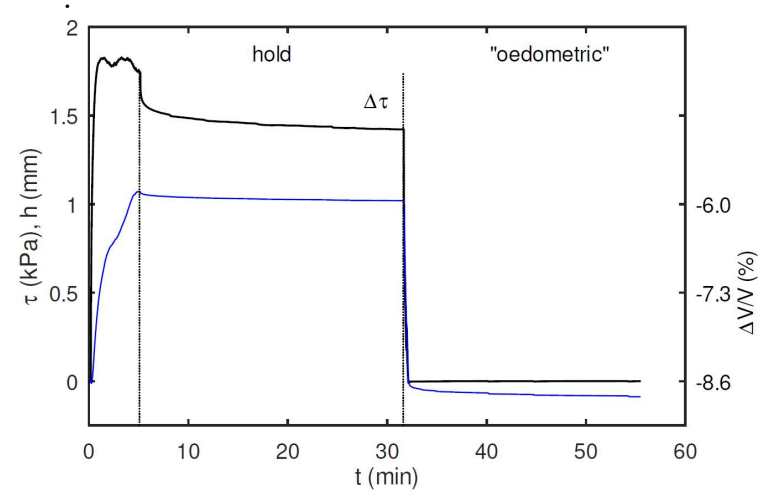

Fig. 1. Shear stress evolution (upper black) and height or relative volume change of the powder bed (lower blue with respectively left or right scale) versus time. Before the holding period, the sample is sheared at constant rotational velocity. After the hold, the shear stress is released to zero. Powder used: coarse torrefied powder (Tor5); $\sigma=1.8 \mathrm{kPa}$; Big cell.

\subsubsection{Relaxation modelling}

For all the experiments performed, the mean long time behaviour (for holding time greater than $100 \mathrm{~s}$, or time delay greater than $100 \mathrm{~s}$ after the release of the shear stress) is observed to be logarithmic. The shear stress signal and/or the height signal $\mathrm{X}$ may be fitted by the expression:

$$
X(t)=X(0)+C_{X} \ln \left(1+t / t_{o, X}\right)
$$

where the origin of the time is chosen at the beginning of the hold (or shear stress release) [12]. This long time logarithmic model usually does not fit the short time behaviour, in particular, $\mathrm{dX} / \mathrm{dt}(0) \neq \mathrm{C}_{\mathrm{X}} / \mathrm{t}_{\mathrm{o}, \mathrm{X}}$. Such relaxation mechanism has already been proved to be a two-step process [13]. The first step is a fast relaxation argued to a single or few particles relaxation process whereas the slow relaxation is the consequence of a collective motion of particles [13].

\section{Results and discussions}

\subsection{Behaviour of the relaxation}

For sake of clarity, only one example of the stress relaxation and bed height during a hold test of a wood powder (Tor5) is presented in Figure 2 in both cells for a normal stress of approximately $10 \mathrm{kPa}$. The two lower curves in black are for $\Delta \tau=\tau-\tau(0)$ and the two upper curves in blue represent $\Delta \mathrm{h}=\mathrm{h}-\mathrm{h}(0)$. The experiment where the hold stands for more than $30 \mathrm{~min}$ is for the smallest cell. The experimental stress data are shown with the fitting curve from eq. (1) - dashed curves in red.

The logarithmic model fits correctly all the data with a correlation coefficient higher than 0.99 . Regarding the stress relaxation, the time $t_{o, \tau}$ is found roughly constant: $0.7 \pm 0.1 \mathrm{~s}$. The coefficient $\mathrm{C}_{\tau}$ is found proportional to the 
normal stress or equivalently to the initial stress $\tau(0)=\mu \sigma$. This indicates that the normal stress applied remains the relevant stress scale for the relaxation process, like for the frictional behaviour.

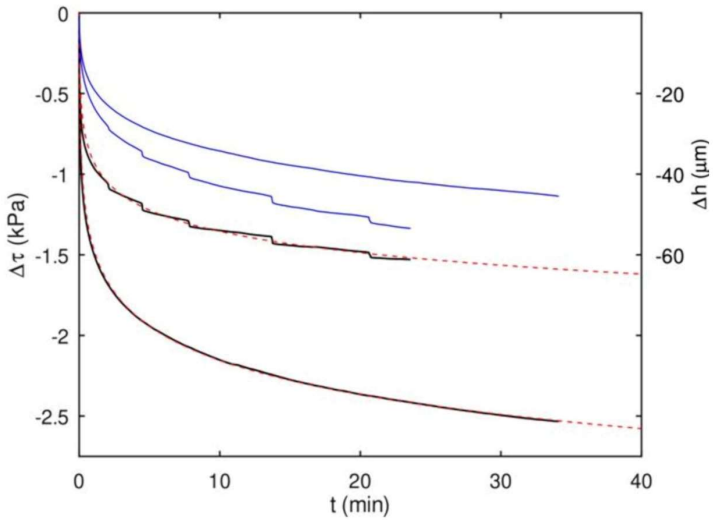

Fig. 2. Shear stress relaxation (two lower curves in black) with their logarithmic fit (dashed red lines) and height decrease (two upper curves in blue) versus holding time. Powder: Tor5. Upper and lower curves: small cell, $\sigma=10.2 \mathrm{kPa}$. Middle curves: big cell, $\sigma=10.5 \mathrm{kPa}$

The relaxation of the shear stress in the small cell is slightly greater than in the big cell. The influence of the aspect ratio of the experimental setup has been previously shown to affect the relaxation behaviour by a different initial density of the powder bed [14], but in this setup, the stiffness and sheared surface are also different.

Regarding the height relaxation, we observe a characteristic time greater than for the stress relaxation, with $t_{o, h}=2.6 \pm 0.3 \mathrm{~s}$. The logarithmic coefficient for height decrease $C_{h}$ is found slightly dependent on the cell with the values of $-19 \pm 2 \mu \mathrm{m}$ and $-25 \pm 3 \mu \mathrm{m}$ for respectively the small and big cell. There is some scattering on $C_{h}$ as exemplified by Figure 3, where the relative height decrease curves do not superpose nor have a monotonous behaviour relatively to the normal stress.

Other powders - Furthermore, the relaxation of the shear stress and the deformation of the powder bed are more important (up to $50 \%$ ) with the powders produced from raw wood. It could be explained by the greater roughness of the particles of raw wood. Miksic et al. [12] showed that the worn beads with flattened asperities display smaller creep deformation or stress relaxation than new beads with significant surface asperities. Lastly, the stress relaxation and the creep deformation are more significant for the coarse powders than for the fine powders. This observation could be explained by the fibrous nature of coarse particles, resulting in increased compressibility [15].

\subsection{Fast relaxation events}

Figure 2 shows the nonmonotonic behaviour of the relaxation of the wood powder in the big cell only. The slow stress relaxation of the granular medium is interrupted by huge relaxation events, which are associated with a corresponding sudden compaction of the powder bed. Such a fast relaxation event, associated with a large reconfiguration of the particle bed, has been previously reported [1,7]. However, the authors mentioned only one isolated event, which had led to a renewal of the logarithmic decay in stress [7].

In the present study, the powder relaxation has been performed during a few dozen of minutes, allowing the observation of recurring large events: the first large event after $2 \mathrm{~min}$., the second after $4 \mathrm{~min}$. and a total of 5 events in the experiment displayed in Figure 2. These several fast relaxation events appear with some regularity, not randomly. The study of the amplitude and temporal occurrence of these large rearrangements has been conducted during the hold or the last part of the procedure, when the shear stress is removed, for which the same phenomena are observed for the height evolution only.

For all the biomass powders used, the staircase shape of the relaxation is observed in the big cell for both signals, but not in the small cell where the shear stress and height decrease smoothly in a logarithmic way. This dependency on the cell size suggests that these large instabilities are triggered by a mechanism which depends on the powder bed apparent stiffness or the size assembly of particles to reconfigure and merits further investigation on its origin.

\subsubsection{Evolution in time}

Figure 3 displays several height decreases under normal stress where 3 or 4 jumps are observed in $25 \mathrm{~min}$. of relaxation. The delay between two consecutive large events increases with the time of relaxation. However, the decrease in height during a large event, $\Delta \mathrm{h}=\delta \mathrm{h}$, and between two events, $\Delta \mathrm{h}=\mathrm{Dh}$, remain mainly constant with the time of relaxation.

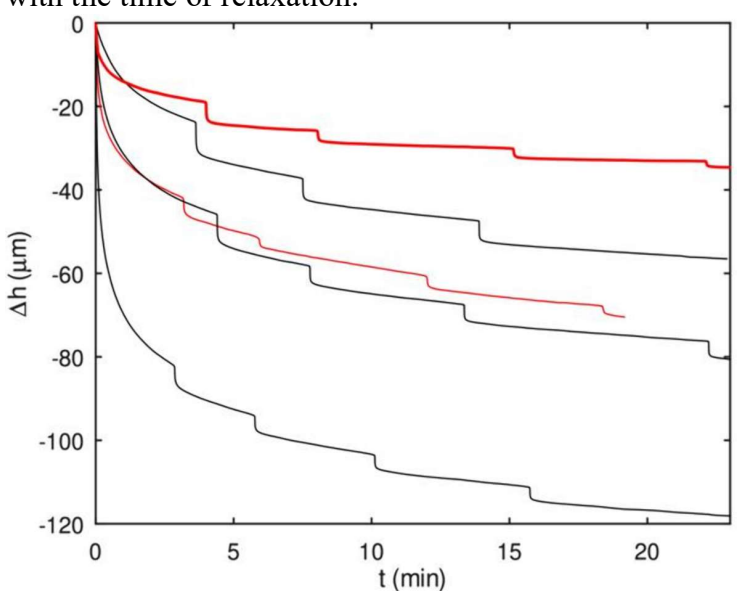

Fig. 3. Height decrease for the coarse torrefied powder (Tor5) for different normal stresses during the last part of the procedure. In red: $\sigma=1 \mathrm{kPa}$. In black, from the top to the bottom, $\sigma=7.0$, 8.8 and $5.3 \mathrm{kPa}$. Thin red line: Initial loose state, thick red line: Initial dense state.

\subsubsection{Influence of the stress history}

The influence of the initial bed density has been observed by repeating the experiment with the lowest normal stress ( $1 \mathrm{kPa}$, red curves in Figure 3) with the initial loose state (thin red line) or with a denser state obtained after shearing under a normal stress of $10.5 \mathrm{kPa}$ (thick red line). 
In the latter case, the height relaxation is lower. Generally, the powder bed displacement between two successive fast relaxation events is much smaller with a dense sample and their occurrence rate is lower. The porosity of the Tor5 sample has decreased from 0.70 in the loose state to 0.65 in the dense state, for the $1 \mathrm{kPa}$ experiments (red curves in Figure 3). It means that the grains are more in contact with each other, making the rearrangements more difficult. For the RawVib sample in this dense state, the fast relaxation events do not occur. This particular behaviour could be due to the fact that this sample is the most spherical and compact sample.

\subsubsection{Influence of the particle size}

For each powder, the mean height jump $\delta$ h and the mean compaction between two jumps Dh are plotted versus the median particle diameter in Figure 4. The mean values are taken over seven experiments performed under the following normal stresses: $0.9,1.8,3.6,5.3,7.0,8.8$ and $10.5 \mathrm{kPa}$.

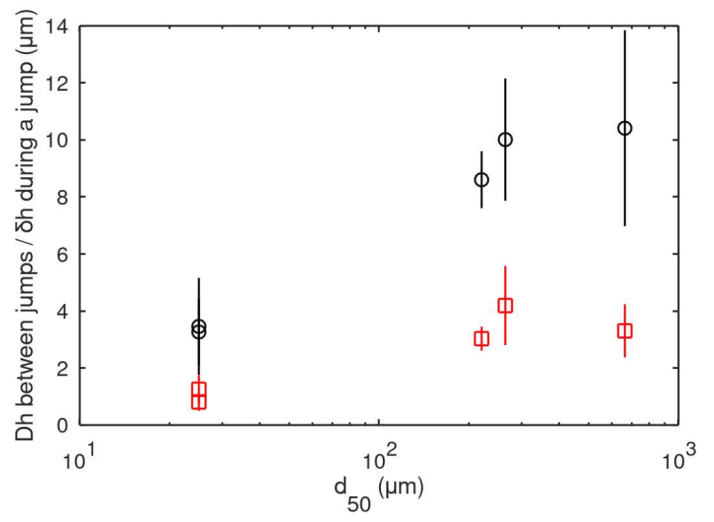

Fig. 4. Bed deformation between two fast relaxation events Dh (black circles), and bed deformation during a fast relaxation event $\delta$ h (red squares) versus the median particle diameter, with associated standard deviations.

It is observed in Figure 4 that $\delta \mathrm{h}$ and $\mathrm{Dh}$ increase with the median of the minimum Feret diameter $d_{50}$. However, this increase is non-linear. It suggests that this particle size is not the only parameter. The amplitude and the occurrence of these sudden rearrangements may depend also on others non-dimensional characteristics of the powders such as the median aspect ratio, the porosity or also the particle size distribution. This dependency still remains to be defined.

Nevertheless, we observe that $\mathrm{Dh}$ and $\delta \mathrm{h}$ are proportional in size, with our different samples. It indicates that they are scaled by the same characteristic length.

\section{Conclusions}

With wood powders, we observe that the logarithmic relaxation or creep may be intermittently interrupted by large events, attributed to sudden rearrangements of particles.
These large events appear for a given deformation and give place to a settlement which is mainly independent of the normal stress. The bed deformation between two large events or during one event increases with the median particle size.

To investigate the origin of that phenomenon, the fibrous character and surface state of the particles have been modified. The preliminary results indicate that only the powder constituted of the most spherical particles in a dense state has a smooth logarithmic relaxation without intermittency.

More investigations are needed to determine the influences of the fibrous character, of the initial density and compressibility of the powder bed, and of the dimensions of the powder bed, observed when changing the size of the sheared powder bed.

The authors thank Göknur Esra Hasbay for her contribution in the experiments. This work was supported by the French Environment and Energy Management Agency (ADEME) and CEA.

\section{References}

[1] R.R. Hartley, R.P. Behringer, Nature 421, 928 (2003)

[2] C. Marone, DM. Saffer, Treatise in Geophysics, chapter IV, Elsevier (2015)

[3] A. de Ryck, R. Condotta, M. Lubert, Eur. Phys. J. E, 11, 159 (2003)

[4] J. Dieterich, Pure Appl. Geophys. 116, 790-806 (1978)

[5] J.R. Rice, A.L. Ruina, J. Appl. Mech. 50, 343 (1983)

[6] A. Amir, Y. Oreg, Y. Imry, PNAS 109, 1850 (2012)

[7] K.A. Murphy, J.W. Kruppe, H.M. Jaeger, Phys. Rev. Lett. 124, 168002 (2020)

[8] C. Vanneste-Ibarcq, T. Melkior, A. de Ryck, EPJ Web of Conferences 140, 08005 (2017)

[9] J. Páchon-Morales, J. Colin, F. Pierre, F. Puel, P. Perré, Biomass Bioenergy 120, 301-312 (2019)

[10] R. Junga, J. Pospolita, P. Niemec, Renew. Energ. 147, 1239-1250 (2020)

[11] ASTM D6773-02, Standard Shear Test Method for Bulk Solids Using the Schulze Ring Shear Tester, ASTM International, West Conshohocken, PA (2002)

[12] A. Miksic, M.J. Alava, Phys. Rev. E 88, 032207 (2013)

[13] J. Brujic, P. Wang, C. Song, D.L. Johnson, O. Sindt, H.A. Makse, Phys. Rev. Lett. 95, 128001 (2005)

[14] O.I. Imole, M. Paulick, V. Magnanimo, M. Morgeneyer, B.E. Chávez Montes, M. Ramaioli, A. Kwade, S. Luding., Powder Technol. 293, 82-93 (2016)

[15] J.E. Rees, K.D. Tsardaka, Int. J. Pharm. 92, 137-141 (1993) 